\title{
Hemiparkinsonism Due to Coiled Posterior Cerebral Artery Aneurysm
}

\author{
Shakya Bhattacharjee, Hrishikesh Kumar, Mona Tiwari, Samput Mallick
}

Can J Neurol Sci. 2013; 40: 101-103

Giant aneurysms involving the posterior cerebral artery (PCA) are rare. Theoretically a giant PCA aneurysm can cause parkinsonian symptom as a result of compression on substantia nigra but there is no actual case report. Few reported cases of aneurysms causing secondary parkinsonism involved middle cerebral artery and vertebral artery. ${ }^{1-5}$ Other commonly known secondary causes of parkinsonism are repeated head trauma, infection, metabolic disorders (Wilson's disease, hypoparathyroidism, pseudohypoparathyroidism), drugs (neuroleptics, Metoclopramide), toxins (1-methyl-1,2,4,6 tetrahydropyridine, carbon monoxide) etc. ${ }^{6}$ Tang et al reported hemiparkinsonism as the presenting feature in a case of primary intracranial hemangiopericytoma. ${ }^{7}$ We present a case of hemiparkinsonism developing after coiling of a large PCA aneurysm.

A 32-year-old female presented with severe headache, vertigo and episodes of diplopia of two months duration. Her fundus was normal and there was no other abnormal neurological sign. At that time she had no tremor, bradykinesia, rigidity or gait problems.

Her routine haematological and serum biochemical parameters were within normal limits. Neuroimaging and angiogram revealed a partially thrombosed saccular aneurysm of $18 \mathrm{~mm} \times 16 \mathrm{~mm} \times 21 \mathrm{~mm}$ arising from $\mathrm{P}_{2}-\mathrm{P}_{3}$ segment junction of left PCA with a narrow neck and mass effect on midbrain at the level of inferior colliculus (Figure 1, Figure 2, supplementary figure). $\mathrm{P}_{4}$ branches of the left PCA were not seen.

Large and giant aneurysms are some of the most challenging vascular pathologies in the central nervous system. Endovascular coil embolization of these lesions is being used increasingly as a safer alternative to surgical clipping and the same was undertaken in this case. ${ }^{8,9}$ A $6 \mathrm{~F}$ guiding catheter was positioned in right vertebral artery via the right femoral route under general anaesthesia followed by positioning of an Excelsior 10-18 microcatheter in the left PCA aneurysm over transcend 14 wire. The $\mathrm{P}_{3}$ segment of the left PCA and aneurysm was occluded with multiple GDC coils. Post embolization left carotid angiogram revealed reformation of the distal branches through the anterior choroidal artery. The PCA was preserved and the aneurysm was almost completely obliterated (Figure 3). She was not considered for steroid therapy before, during or after the operation.

After four weeks of endovascular coiling, she developed rest tremor of her right hand and started dragging her right leg while walking. She also complained of slowing down in all her motor activities. She had no complaint of diplopia or blurring of vision. All these motor symptoms were insidious in onset and progressed with time. On examination, she had rest tremor,

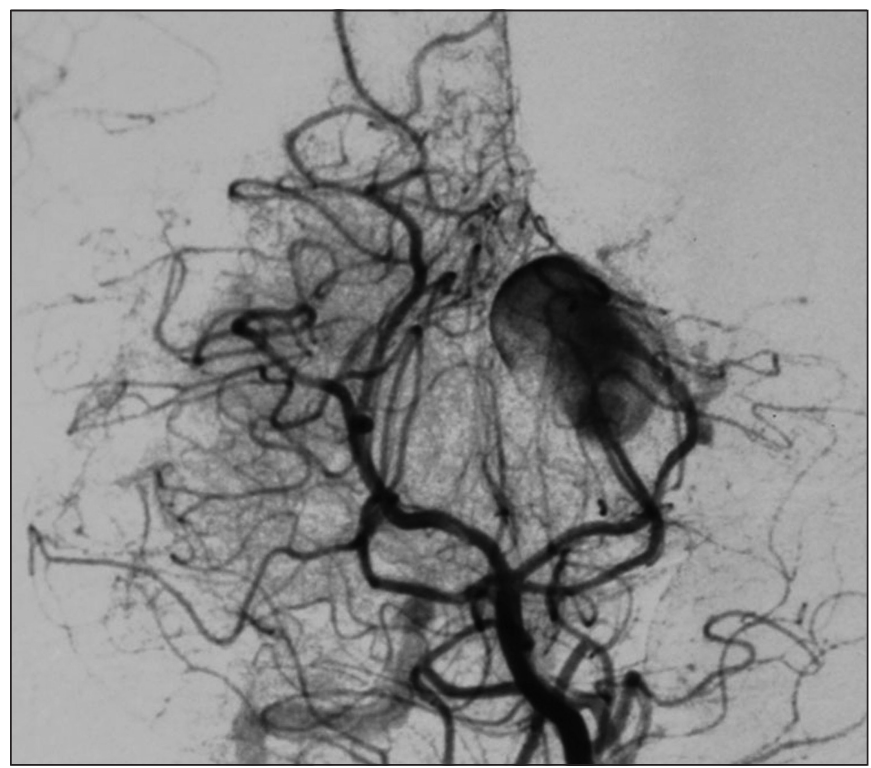

Figure 1: Digital subtraction angiogram (DSA) AP view showing a partially thrombosed saccular aneurysm of PCA before endovascular coiling

bradykinesia (Video 1 on-line) and rigidity involving right upper and lower limbs. The tremor was restricted to the right hand. The tremor was a rest tremor, had no kinetic component. Moreover the tremor demonstrated the re-emergence phenomenon. She had no signs of cortical spinal tract involvement. Her cranial nerve examinations, including ocular movement, were found to be normal. Romberg's sign was negative and there was no cerebellar sign.

The patient also developed hydrocephalus following the endovascular coiling. Her hydrocephalus was initially considered to be the cause of the above symptoms of parkinsonism as hydrocephalus is a well established cause of

From the Institute of Neurosciences (HK, MT, SM); Department of General Medicine Peerless Hospital (SB), Kolkata, India.

Received May 10, 2012. Final Revisions Submitted July 27, 2012.

Correspondence to: Shakya Bhattacharjee, 95/B, Indra Biswas Road, Kolkata-700037, West Bengal, India. Email: shakyadoc@rediffmail.com. 


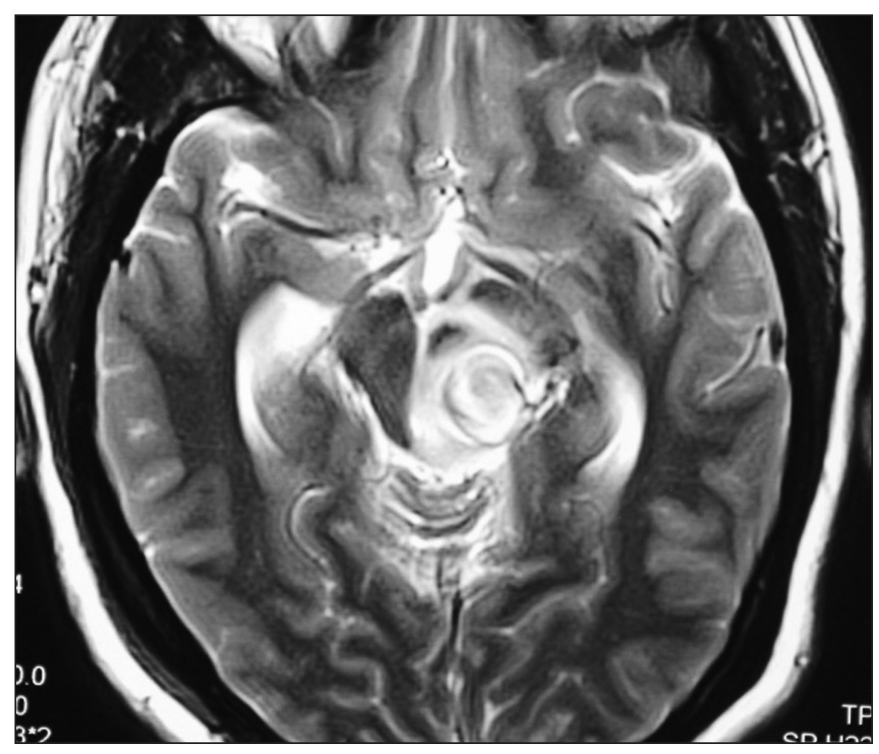

Figure 2: MRI brain showing significant midbrain compression because of a partially thrombosed aneurysm before coiling .

parkinsonism. She underwent ventriculo-peritoneal shunt operation to reduce the same. The hydrocephalus improved significantly but her hemiparkinsonian symptoms (both rest tremor and bradykinesia) persisted.

A repeat computed tomogram (CT) scan brain revealed slight increase in the size of the coiled aneurysmal sac. The aneurysm had a size of $22 \mathrm{~mm} \mathrm{X} 21 \mathrm{~mm} \mathrm{X} 22 \mathrm{~mm}$ and was still saccular in shape. (Figure 4) It was exerting significant mass effect at the midbrain but no edema was noted.

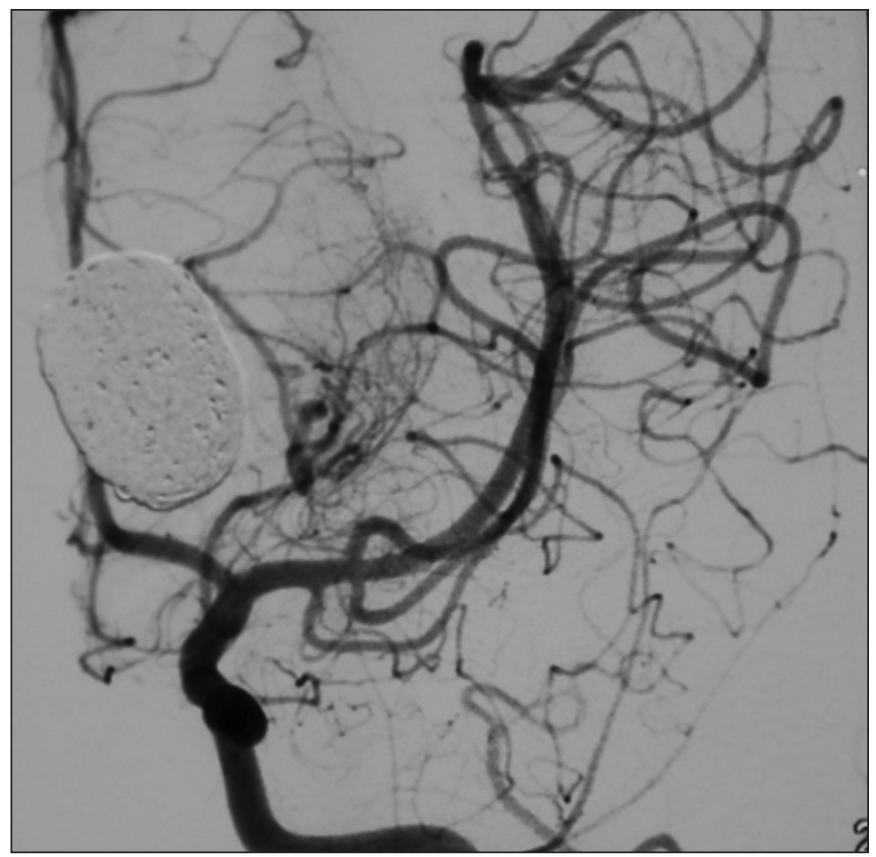

Figure 3: Successful obliteration of the aneurysm immediately after endovascular GDC.
Mordasini et al found more than $47 \%$ recanalisation rate in posterior cerebral circulation aneurysm treated with 3D GDC and aneurysm size did not have any correlation with the recanalisation rate. The use of 3D GDC yielded significantly high early occlusion rates in large aneurysms but did not improve the incidence of recanalisation rate. ${ }^{9}$ Surgical options like further endovascular coiling, ligation, endovascular parent vessel sacrifice, surgical trapping and bypass, and endovascular flow diversion were discussed but the patient and her relatives denied any further intervention because of peri and post -operative morbidity issues despite the potential threat of rebleeding and further mass effect in the future. Trihexyphenidyl in the dosage of $2 \mathrm{mg}, 8$ hourly was started. After two weeks of starting trihexyphenidyl, the patient's symptoms improved significantly. Her tremor was much better, bradykinesia had almost disappeared (See Video 2 on-line) and gait was also improved. At one year of regular follow-up to date she is doing well on anticholinergic treatment.

Secondary parkinsonism resulting from unruptured giant or large intracerebral aneurysms or related to treatment of the aneurysm is rare. Two separate cases of giant middle cerebral artery (MCA) aneurysm resulting in parkinsonian symptoms were reported by Gross and Bostantjopoulou et al respectively. ${ }^{1,4}$ In both cases parkinsonism improved after surgical excision of aneurysm. Another case of internal carotid artery aneurysm resulting in asymmetric parkinsonism was reported but the symptoms did not improve after internal carotid artery (ICA) ligation. ${ }^{2}$ Krause et al recorded a case of levodopa responsive hemiparkinsonism due to a midbrain Virchow robin space but there was no mention of aneurysm in that case. ${ }^{10}$ A case of subarachnoid hemorrhage who developed symptoms of parkinsonism after clipping of bilateral aneurysms of MCA was reported by Jaskólski et al. ${ }^{3}$ Choudhury et al reported hemiathetosis in a child following middle cerebral artery aneurysm. ${ }^{11}$ Juvenile parkinsonian hemi-syndrome in a case of

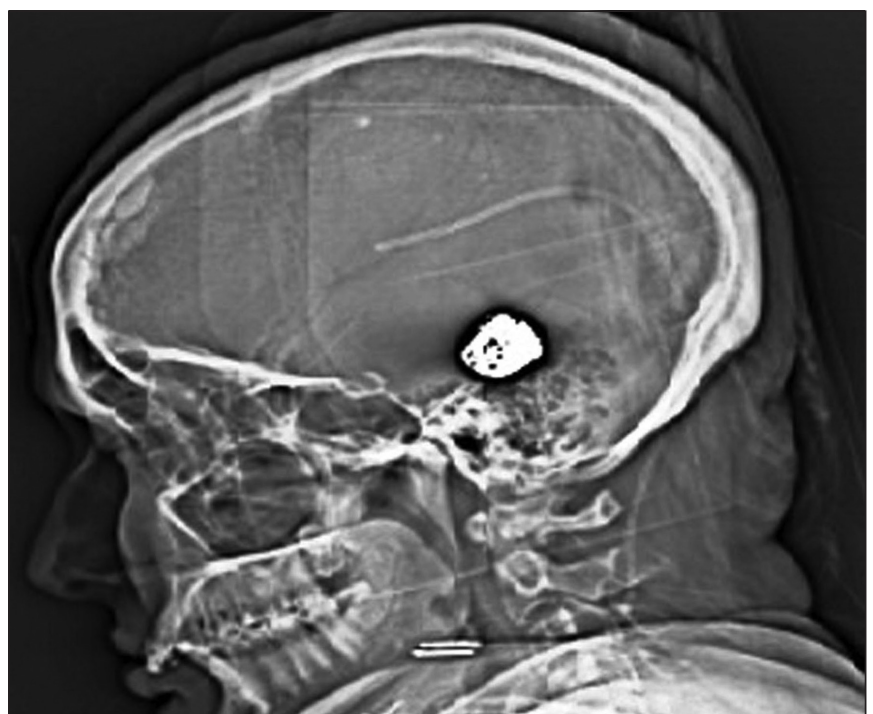

Figure 4: Recent Scannogram showing shunt tube and coiled aneurysm that has increased in size. 
extracranial arteriovenous aneurysm of the vertebral artery was reported by Bossi and Caffaratti. ${ }^{5}$

None of the above cases mentioned development of parkinsonism in a case of PCA aneurysm. The present patient is the first case report of developing parkinsonism in a patient with a PCA aneurysm. In the present case the parkinsonian symptoms developed after four weeks of endovascular coiling and the symptoms gradually progressed. The possible mechanism could be the compression of the midbrain by the aneurysmal sac leading to nigrastriatal neuronal degeneration or altered perfusion. ${ }^{2,6}$

Our patient had tremor that was predominantly unilateral right upper limb parkinsonism but hydrocephalus causes mainly bradykinesia predominantly, bilateral lower body parkinsonism. Response to trihexyphenidyl in hydrocephalus induced parkinsonism is not known. The persistence of the tremor and bradykinesia after improvement of the hydrocephalus makes left half of the midbrain compression the likely reason for right sided hemiparkinsonism instead of hydrocephalus.

The exact cause of the growth of the aneurysm was difficult to determine but in our patient no migration of the coil or recanalisation was found. Formation of GDC-thrombus complex is the most likely explanation but influence of hemodynamic stress (water hammer effect), hemorrhage from vaso vasorum into the aneurysmal wall could also be responsible for the increase in the volume of the aneurysmal sac after endovascular therapy. ${ }^{12,13}$ There was one case report of perioperative and histological diagnosis of vasa-vasorum as the cause of regrowth of vertebral artery aneurysm even after complete endovascular occlusion. ${ }^{13}$

The strictly lateralized symptoms and contralateral abnormalities in the midbrain suggest a possible relationship between the midbrain aneurysm and hemiparkinsonism in the presented case. Though Holmes or rubral tremor was possible

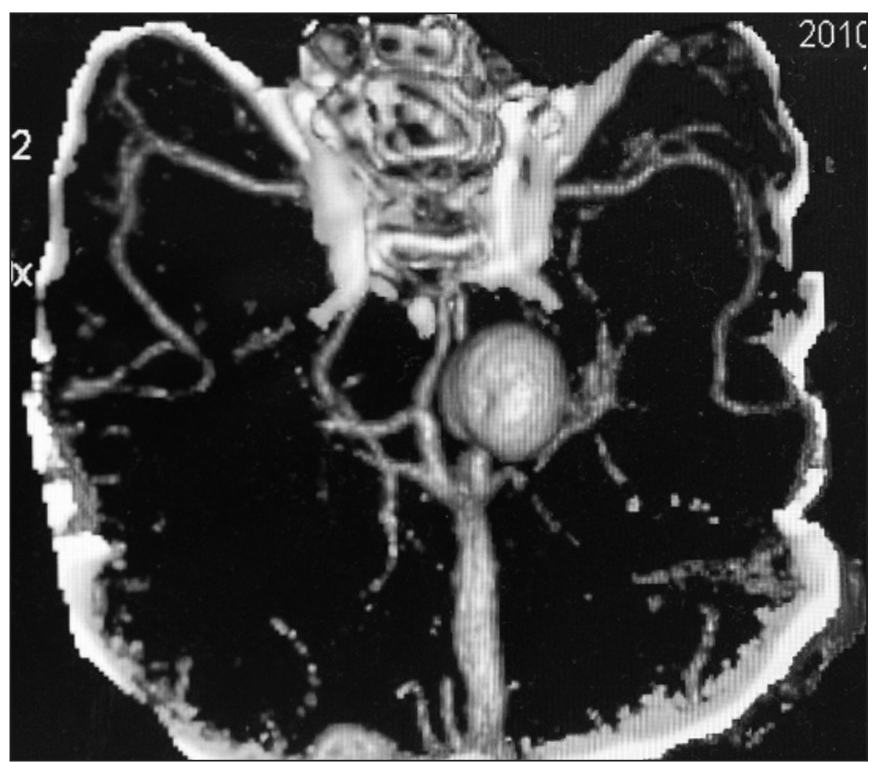

Supplementary: Angiogram with $3 D$ reconstruction showing narrow necked saccular aneurysm because of midbrain compression, the patient did not have postural or action tremor and the tremor was predominantly distal which is unlikely in a case of rubral tremor. Moreover isolated midbrain pathology was rarely described as a cause of rubral (midbrain) tremor. Thalamic involvement in the form of infarction or hemorrhage was found to be the commonest cause. A parallel dysfunction of cerebello-thalamic and nigrostriatal pathways seems to be a prerequisite to produce this tremor. ${ }^{14}$

Considering the young age and tremor predominant parkinsonian symptoms, trihexyphenidyl was started and the response was excellent. To the best of our knowledge, this is probably the first case where aneurysm induced parkinsonian symptoms responded to medications. The presented case also highlights that endovascular coiling of a giant PCA aneurysm can result in parkinsonian symptoms as a result of mass effect.

\section{REFERENCES}

1. Gross M. Giant middle cerebral aneurysm presenting as hemiparkinsonism. J Neurol Neurosurg Psychiatry. 1987;50: 1075.

2. Sibon I, Rajabally Y, Tison F. Parkinsonism as a result of a giant aneurysm. Mov Disord. 1999;14:159-61.

3. Jaskólski D, Zawirski M, Niewodniczy A. Parkinsonism after clipping of bilateral aneurysms of the middle cerebral arteries. Zentralbl Neurochir. 1989;50:193-5.

4. Bostantjopoulou S, Katsarou Z, Petridis A, Andreou A. Aneurysm presenting as parkinsonism. Neurology. 2006;67:2028.

5. Bossi L, Caffaratti E. Juvenile parkinsonian hemi-syndrome in a case of extracranial arteriovenous aneurysm of the vertebral artery. Clinical and radiological study. Minerva Radiol. 1967;12: 533-8

6. Mahlon RD, Jorge LJ. Parkinson's disease and other movement disorders. In: Kasper DL, Fauci AS, Longo DL, et al, editors. Harrison's principles of internal medicine. New York: McGraw Hill; 2005. p. 2408.

7. Tang V, Woulfe J, Grimes D. Primary intracranial hemangiopericytoma presenting as hemiparkinsonism. Can J Neurol Sci. 2011;38:349-51

8. Yue W. Endovascular treatment of unruptured intracranial aneurysms. Interv Neuroradiol. 2011;17:420-4.

9. Mordasini P, Schroth G, Guzman R, Barth A, Seiler RW, Remonda L. Endovascular treatment of posterior circulation cerebral aneurysms by using Guglielmi detachable coils: a 10-year single-center experience with special regard to technical development. Am J Neuroradiol. 2005;26:1732-8.

10. Krause M, Hahnel S, Haberkorn, Meinck HM. Dopa responsive hemiparkinsonism due to midbrain Virchow Robin spaces. J Neurol. 2005;252:1555-7.

11. Choudhury AR, al Amiri NH, al Moutaery KR, Aabed M, Strelling MK.Giant middle cerebral aneurysm presenting as hemiathetosis in a child and its spontaneous thrombosis. Childs Nerv Syst. 1991:7:59-61.

12. Tomokiyo M, Kazekawa K, Onizuka M, et al. Mechanisms of perianeurysmal edema following endovascular embolization of aneurysms. Interv Neuroradiol. 2007;13(s1):145-50.

13. Iihara K, Murao K, Sakai N, et al. Continued growth of and increased symptoms from a thrombosed giant aneurysm of the vertebral artery after complete endovascular occlusion and trapping: the role of vasa vasorum. J Neurosurg. 2003;98: 407-13.

14. Gajos A, Bogucki A, Schinwelski M, et al. The clinical and neuroimaging studies in Holmes tremor. Acta Neurol Scand. 2010;122: 360-6 